\title{
Purtscher's Retinopathy after Airbag Injury, Six month Progression and Sequelae
}

\author{
Ridha F*, Chin Loy K, Lopez-Beachamp C and Khan B \\ Department of Ophthalmology, Howard University Hospital, USA \\ *Corresponding author: Dr Faisal Ridha, Department of Ophthalmology, Howard University Hospital, Washington, DC, USA
}

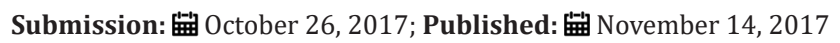

\section{Introduction}

Purtscher's retinopathy is a microvasculopathy that has been well documented but poorly understood. The earliest case was reported by Otmar Purtscher in 1910, which described a man who suffered cranial trauma after falling out of a tree. The current incidence of Purtscher's retinopathy is estimated at 0.24 persons per million per year [1]. When identified, Purtscher's is most commonly seen in young men in the setting of trauma [2]. Seat belt trauma associated with motor vehicle accidents leading to Purtscher's retinopathy has been reported in several papers. However, only one previous case of Purtscher's retinopathy associated with traumatic optic neuropathy has been attributed to airbag injury [3-7].

\section{Case Report}

We report a case of a 30 year old male who was the restrained front seat passenger involved in a motor vehicle accident with airbag deployment. The patient reported being struck directly in the region of the right brow by the airbag resulting in small eyebrow laceration. CT scan of the head was normal and there were no signs of seat belt injury. In the emergency department, he complained of monocular blurred vision and photopsias. Near visual acuity was $20 / 40+2$ in the right eye and $20 / 20$ in the left with normal anterior segment examination. The fundus exam showed poorly localized areas of whitening within the peripapillary retina which was initially mistaken for commotio retinae accompanied by a blunted foveal reflex. He otherwise had normal appearing optic nerve, vitreous, and vessels. Subsequent OCT of the macula showed significant sub retinal and intraretinal fluid in the right eye with central pigment epithelium detachment.

The patient followed up 3 days later complaining of para central scotoma and visual acuity of 20/30 in the right eye. At this time, the dilated exam revealed pronounced, peripapillary yellow-white patches consistent with Purtscher flecken and cotton wool spots in the right eye only. There was also an intraretinal hemorrhage along the superior arcade and an abnormal foveal reflex in the affected eye. With this constellation of findings, Purtscher's retinopathy was diagnosed and the decision was made to observe for clinical improvement. Given the clinical diagnosis, fluorescein angiography was deferred.
At six month follow up, the patient's visual acuity had returned to $20 / 20$ with complete resolution of the intraretinal lesions. However, he complained of a persistent para central scotoma which was demonstrated using Amsler grid, and confirmed with 10-2 Humphrey Visual Field. OCT of the right eye showed a small area of sub foveal RPE irregularity despite resolution of the macular edema.

\section{Discussion}

Purtscher's retinopathy is diagnosed clinically with the presence of three out of the five following criteria as adopted by Miguel and colleagues [1].

a. Purtscher flecken, (pathognomonic but reported to occur in only $50 \%$ of cases).

b. Retinal hemorrhages (reported in $83-92 \%$ of cases).

c. Cotton-wool spots (typically restricted to the posterior pole).

d. Probable or plausible explanatory etiology.

e. Complementary investigation compatible with diagnosis.

In their review, the two most frequent etiologies were trauma and acute pancreatitis. Other associations include fat embolism syndrome, renal failure, childbirth, connective tissue disorders, and other rare etiologies [8]. The acute findings and visual acuity typically improve without treatment in the months after the inciting event. Though there are several theories with regard to the mechanism, the majority of evidence points to an embolic occlusion of the precapillary retinal arterioles [1,8]. One theory cites the micro-embolization of fat and pancreatic proteases leading to precapillary retinal occlusion and micro vascular infarcts. Another proposed mechanism involves leukoembolization related to leukocyte aggregation and the C5 complement pathway activation. Other studies have discussed capillary endothelial damage or increased intracranial pressure as contributing factors, though the true mechanism is not well understood.

While injuries secondary to airbag deployment are frequently encountered, mortality has continued to improve with these safety 
measures, and ocular injuries tend to be less severe [9]. Although our patient was restrained in this case, we suspect the impact of the airbag to be the primary mechanism of injury as there were no signs or symptoms of chest trauma. Our case highlights the classic features of the condition with clinical improvement and extended follow up. Our patient demonstrated the Purtscher flecken and cotton wool spots localized to the posterior pole, intraretinal hemorrhages, and probable etiology. Rapid improvement of the macular edema correlated closely with the improvement in visual acuity. Despite this, the patient had a permanent paracentral scotoma consistent with damage in the outer segments of the photoreceptors. These OCT findings and persistent scotomas are supported by other cases in the literature [1,3,10-12].

The need for well documented cases was reiterated by Miguel et al in their review of the literature; only $1 / 3$ of the cases reviewed in their study had reported visual acuity beyond one month. Although several case reports have suggested potential benefits of steroid treatment, Miguel et al found no significant improvement. However, it has been proposed that the duration of the acute retinal changes, particularly retinal edema, may serve as an important prognostic factor for long term sequelae [12]. Treatment aimed at early reduction of retinal edema in subsets of patients may demonstrate better long term outcomes if future trials are able to be carried out

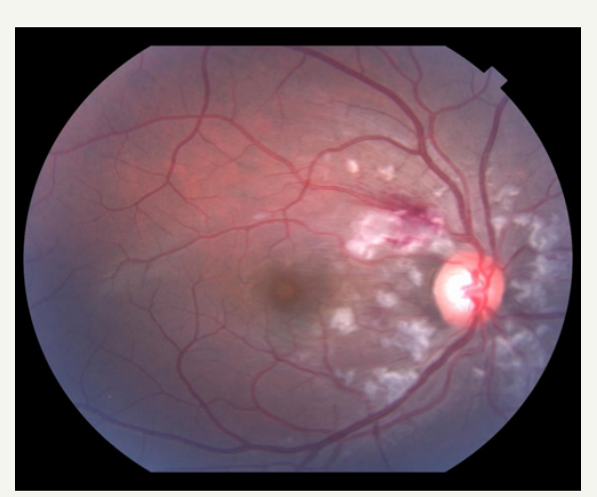

Figure 1A: Fundus photo right eye 3 days after the accident showing polygonal white lesions in a peripapillary distribution, and area of intraretinal hemorrhage along the superior arcade.

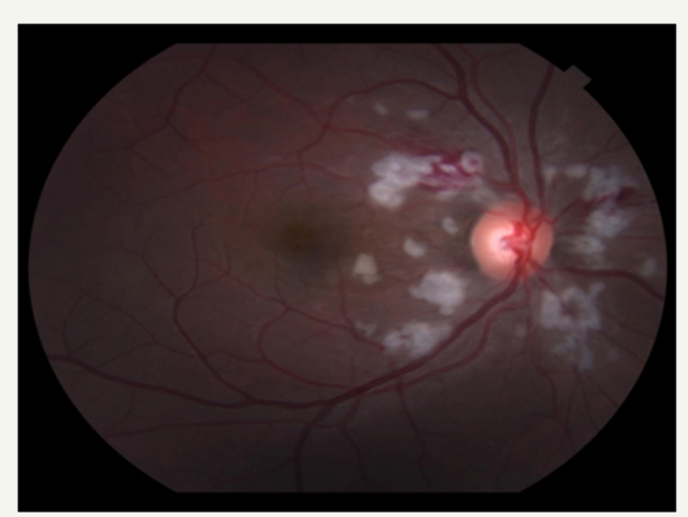

Figure 1B: Fundus photo of the right eye 10 days after the accident showing similar, more defined white lesions.
In conclusion, this report describes a case of unilateral Purtscher's retinopathy secondary to direct airbag injury. Despite improved visual acuity, the patient had a permanent paracentral scotoma more than six months after the injury. Purtscher's is a rare finding that should be considered in cases of airbag injuries, with potential long term visual sequelae (Figures 1A-1D, 2A-2B, 3 \& 4).

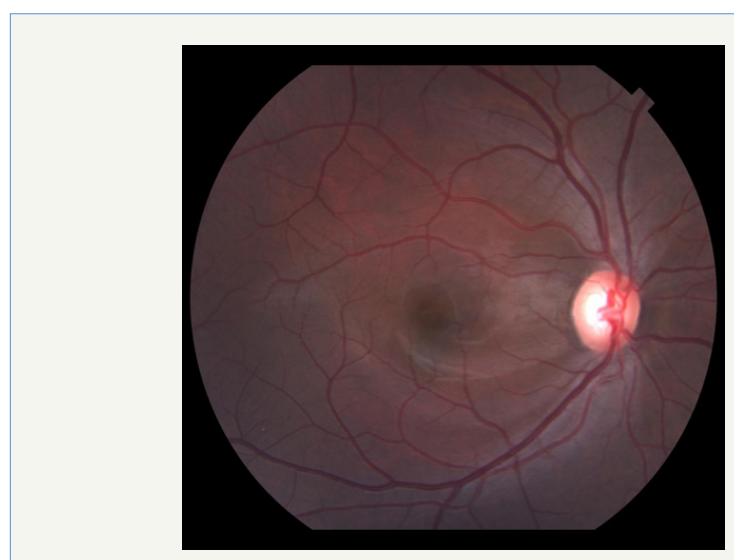

Figure 1C: Fundus photo of the right eye at 6 month follow up showing complete resolution.

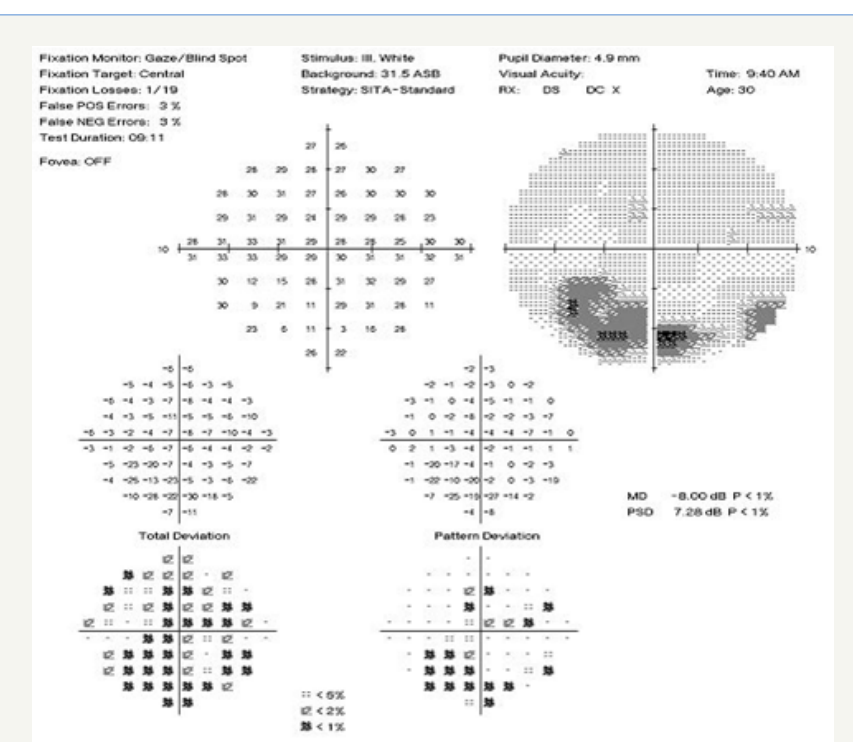

Figure 1D: 10-2 Humphrey Visual Field at 6 month follow up showing persistent paracentral scotoma.

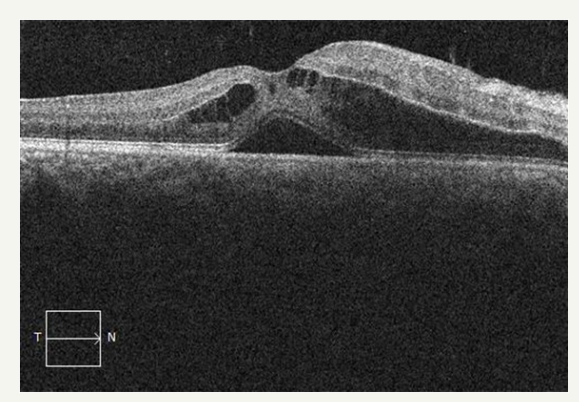

Figure 2A: OCT OD a few hours after the accident, showing significant intraretinal and subretinal fluid within the macula. 


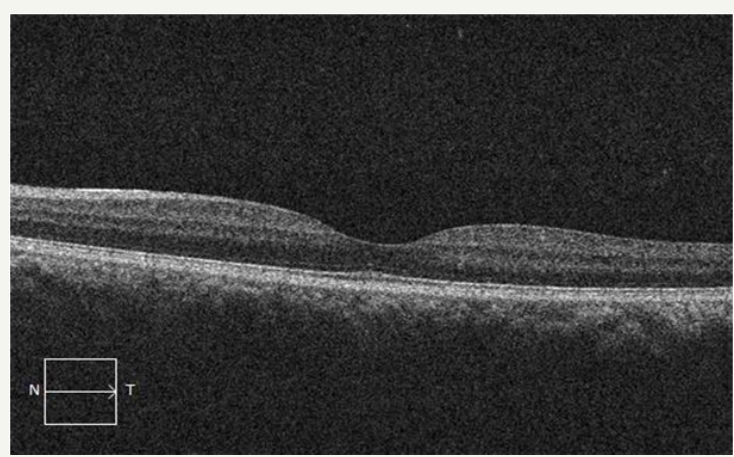

Figure 2B: OCT OS with normal retinal anatomy.

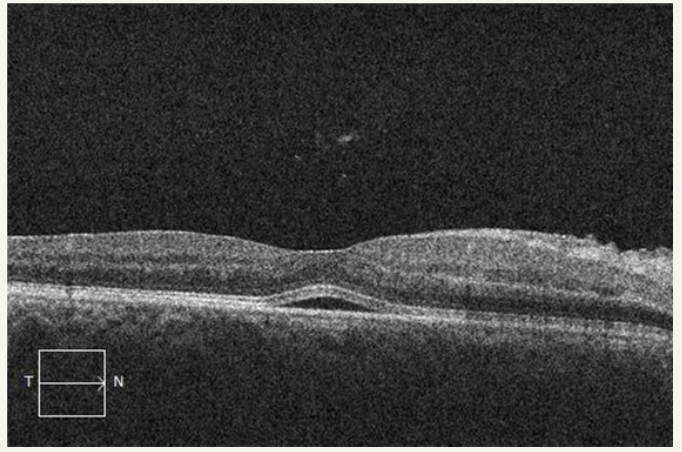

Figure 3: OCT OD at day 3 of follow up showing decreased intraretinal fluid and pigment epithelial detachment.

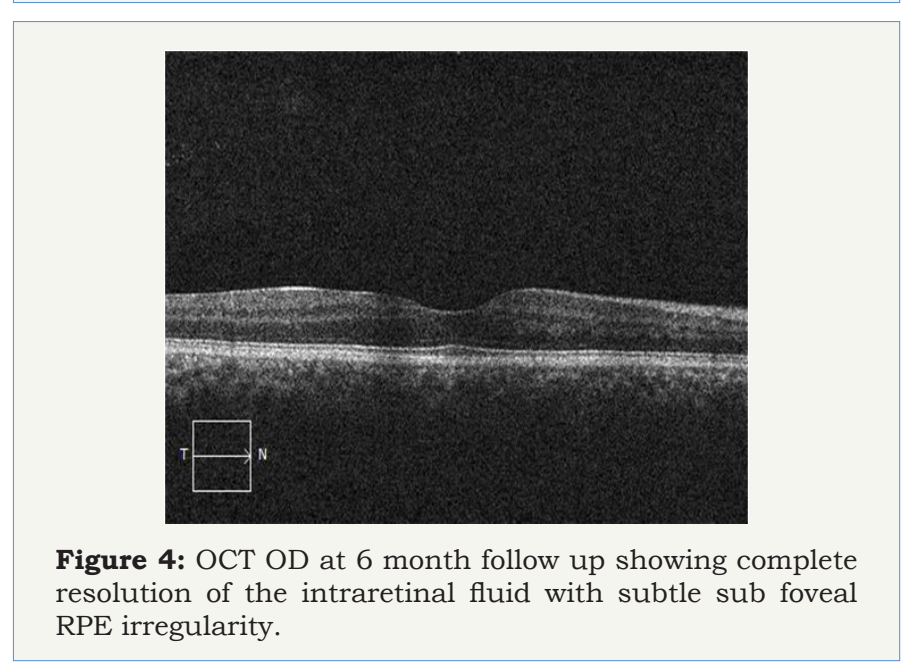

\section{References}

1. Miguel AI, Henriques F, Azevedo LF, Loureiro AJ, Maberley DA (2013) Systematic review of Purtscher's and Purtscher-like retinopathies. Eye 27(1): 1-13.

2. Agrawal A, McKibbin M (2007) Purtschers retinopathy: epidemiology, clinical features and outcome. Br J Ophthalmol 91(11): 1456-1459.

3. Hoare GW (1970) Traumatic retinal angiopathy resulting from chest compression by safety belt. Br J Ophthalmol 54(10): 667-669.

4. Kelley JS (1972) Purtschers retinopathy related to chest compression by safety belts: fluorescein angiographic findings. Am J Ophthalmol 74(2): 278-283.

5. Lindenblatt B, Hinzpeter EN (1984) Traumatic retinal angiopathy (Purtscher)-an early case report. Fort schr Ophthalmol 81: 228-230.

6. Archer DB, Earley OE, Page AB, Johnston PB (1988) Traumatic retinal angiopathy associated with wearing of car seat belts. Eye 2(6): 650-659.

7. Shah GK, Penne R, Grand MG (2001) Purtschers retinopathy secondary to airbag injury. Retina 21(1): 68-69.

8. Agrawal A, Mckibbin MA (2006) Purtschers and Purtscher-like retinopathies: a review. Surv Ophthalmol 51(2): 129-136.

9. Almahmoud T, Barss P (2014) Vehicle occupant restraint systems impact on eye injuries: a review. Surv Ophthalmol 59(3): 334-344.

10. Soliman W, Zibrandtsen N, Jorgensen T, Sander B, Alsbirk PH, et al. (2007) Sequels of Purtscher's retinopathy imaged by enhanced optical coherence tomography. Acta Ophthalmol Scand 85(4): 450-453.

11. Gil P, Pires J, Costa E, Matos R, Cardoso MS, et al. (2015) Purtscher retinopathy: to treat or not to treat? Eur J Ophthalmol 25(6): 112-115.

12. Holak HM, Holak S (2007) Prognostic factors for visual outcome in Purtscher retinopathy. SurvOphthalmol 52(1): 117-118. 